\title{
Plasma Levels of Retinol, Retinol-binding Protein, all-trans Beta-Carotene and Cryptoxanthin in Low Birth Weight Infants
}

\author{
Hideki Moji,* Takushi Murata, Takao Morinobu, \\ Mitsuhiro Manago, Hiroshi Tamai, Ryozo OKamoto, \\ Makoto Mino, Masanori Fujimura, ${ }^{1}$ \\ and Toru TAKEUCHI ${ }^{1}$ \\ Department of Pediatrics, Osaka Medical College, \\ Takatsuki 569, Japan \\ ${ }^{1}$ Department of Neonatology, Osaka Medical Center and \\ Research Institute for Maternal and Child Health, \\ Izumi 590-01, Japan
}

(Received July 29, 1995)

\begin{abstract}
Summary In neonatal medicine, it is thought that retinol is useful for preventing CLD and for fetal development. However, $\beta$-carotene and other vitamin A precursors have not been studied in neonates with CLD or other disorders. Cord blood of neonates including ELBW and VLBW infants was assayed for plasma levels of retinol, RBP, $\beta$-carotene and cryptoxanthin. Plasma $\beta$-carotene levels in ELBW and VLBW were lower than that in term infants, but plasma cryptoxanthin levels in ELBW and VLBW were about the same as in term infants. Plasma retinol and RBP levels showed almost same levels during 23-41 gestational weeks. Maternal smoking reduced plasma $\beta$-carotene but not cryptoxanthin, retinol, or RBP levels. IUGR was associated with increased cryptoxanthin levels in cord blood. Serious neonatal diseases, including CLD and ROP manifested no significant effects on the cord blood vitamin levels. Thus, the occurrence of these diseases at birth could not be predicted by examination of vitamin levels in cord blood.
\end{abstract}

Key Words retinol, $\beta$-carotene, cryptoxanthin, neonate, IUGR, toxemia, chronic lung disease, low birth weight infant

Although many extremely low birth weight (ELBW) and very low birth weight (VLBW) infants can now survive due to advanced medical care and the development of mechanical ventilators and monitoring equipment for neonates, as well as the use of surfactants, a new problem has arisen as regards chronic lung disease (CLD). The relation of CLD to vitamin A (retinol) nutritional status has

* To whom correspondence and reprint requests should be addressed. 
been investigated because retinol plays an active role in the orderly growth and differentiation of epithelial cells including the lining cells in the respiratory tracts in neonates $(2,4,14)$. Hustead et al. (13) and Shenai et al. (3) have reported that plasma retinol levels were usually depressed in infants with CLD. However, the exact reasons for the low plasma retinol levels are still obscure. Therefore, it is important to investigate the retinol, retinol-binding protein (RBP) as well as carotenoids which is the precursor of retinol. To our knowledge, only a few reports have appeared concerning carotenoids, vitamin A precursors including $\beta$-carotene, and cryptoxanthin in infants as an index of vitamin nutrition (15). In the present study, the plasma levels of retinol, all-trans $\beta$-carotene, cryptoxanthin and RBP in the cord blood of ELBW and VLBW infants were investigated in relation to various attributes of mothers and neonates.

\section{MATERIALS AND METHODS}

Subjects and sample collection. Cord blood was collected at birth via the umbilical vein from 96 infants, comprising 45 females and 51 males, in the Osaka Medical Center and Research Institute for Maternal and Child Health during the period from January 1990 to December 1992. The birth weight of the infants ranged from $554 \mathrm{~g}$ to $3,558 \mathrm{~g}$ and their gestational age from 23 to 41 weeks. The group included 18 small-for-date infants and 20 infants with postnatal CLD. No major congenital anomalies were present in any of the infants (Table 1).

Nineteen pregnant women receiving prenatal care at Osaka Medical College, were recruited from June 1994 through April 1995, comprising 11 normal pregnant women without detected hypertension, proteinuria, or edema during gestation, and 8 patients with toxemia but without any family or personal history of hypertension, hydramnions, or diabetes. These subjects were studied after 28 weeks of gestation. The toxemia was diagnosed in accordance with the definition of the Japan Society of Obstetrics and Gynecology. Blood samples were collected from the 36th to the

Table 1. Demographic and physical characteristics of study subjects $(n=96)$.

\begin{tabular}{lc}
\hline \multicolumn{1}{c}{ Characteristics } & Values* \\
\hline Gestational age (weeks) & $30.8 \pm 4.7(23.1-41.2)$ \\
Birth weight (g) & $1,458 \pm 743(544-3,558)$ \\
Male/female & $51 / 45$ \\
IUGR & 18 \\
Chronic lung disease & 20 \\
Mother with toxemia & 12 \\
Mother with IUI & 40 \\
Mother with smoking habit & 11 \\
Mother with drinking habit & 12 \\
*Plus \pm minus values are M \pm SD (range); other values are absolute number of \\
patients. IUGR, intrauterine growth retardation; IUI, intrauterine infections.
\end{tabular}


41st gestational weeks. The study protocol was approved by the Ethics Committees of the hospitals and informed consent was obtained from the parents.

Sample preparations. The plasma was separated by centrifuging at 3,000 rpm immediately after blood collection, and was stored at $-80^{\circ} \mathrm{C}$ until assay.

Chromatography. The HPLC system for cryptoxanthin and AT-BC was comprised of a pump system P-530, a column heater T-80, an amperometric detector $\Sigma 875$, and a sample injector $\Sigma 80$ (all from IRIKA Co. Ltd., Kyoto, Japan) and a chromatopac C-R6A (Shimadzu, Kyoto, Japan). The mobile phase was methyl alcohol: dichloromethane $=90: 10$, containing $50 \mathrm{~mm} \mathrm{NaClO}_{4}$ (all HPLC grade, Nacalai Tesque, Inc., Kyoto, Japan). The column for cryptoxanthin and AT-BC; Hibar LichroCART, Superspher $100 \mathrm{RP}-18$ (e) $(4 \mu \mathrm{m}) 244 \mathrm{~mm} \times 4 \mathrm{~mm}$ (Cica-MERCK, Darmstadt, Germany); flow rate $0.8 \mathrm{ml} / \mathrm{min}$; injection volume of sample $50 \mu$. The HPLC system for retional was comprised of a pump system LC5A, fluorescence HPLC monitor RF-550 (all from Shimadzu), an auto loader $\Sigma 986$ (IRIKA) and a chromatopac C-R6A (Shimadzu). The mobile phase was ethyl alcohol: $\mathrm{H}_{2} \mathrm{O}=95: 5$ (all HPLC grade, Nacalai Tesque, Inc.). The column for retinol: QC PACK ODS $(4.6 \mathrm{~mm} \times 250 \mathrm{~mm}$, IRIKA); flow rate $1.0 \mathrm{ml} / \mathrm{min}$; injection volume of sample $20 \mu \mathrm{l}$. The concentrations of the authentic AT-BC, cryptoxanthin and retinol were determined with a Hitachi U-2000 spectrophotometer (Hitachi Co. Ltd., Tokyo, Japan) using molecular coefficients $\mathrm{E}_{\mathrm{cm}}^{\%}=2,620$ at $453 \mathrm{~nm}$ and $\mathrm{E}_{\mathrm{cm}}^{\%}=2,370$ at $452 \mathrm{~nm}$, and $\mathrm{E}_{\mathrm{cm}}^{\%}=1,835$ at $325 \mathrm{~nm}$ in ethyl alcohol. The Mann-Whitney U-test and regression analysis were used for statistical analysis (StatView 4, Abacus Concepts Inc, Berkeley, CA, USA). The molar ratios of retinol, cryptoxanthin, AT-BC and RBP were calculated from plasma concentrations, assuming molecular weights of 286, 553, 534, and 21,000, respectively.

\section{RESULTS}

Plasma levels of $\beta$-carotene, cryptoxanthin, retinol and $R B P$ in cord blood in relation to gestational age

The plasma levels of AT-BC, cryptoxanthin, retinol and RBP in cord blood in relation to gestational age are shown in Figs. 1 to 3. AT-BC levels in cord blood were significantly correlated with gestational age $(r=0.428, p<0.001)$ (Fig. 1), while cryptoxanthin levels were not (Fig. 2). Plasma retinol and RBP levels (Figs. 3-A and B) were also not correlated with gestational age.

Relation of cord blood levels to maternal attributes including smoking habits and toxemic states

Cord plasma levels were also considered in relation to smoking habits of mothers. AT-BC levels in the cord blood of neonates whose mothers were smokers were significantly lower as compared with those whose mothers were nonsmokers, while cryptoxanthin, retinol and RBP levels in cord blood were not influenced by the mother's smoking habits (Fig. 4). Cryptoxanthin levels in cord blood of 


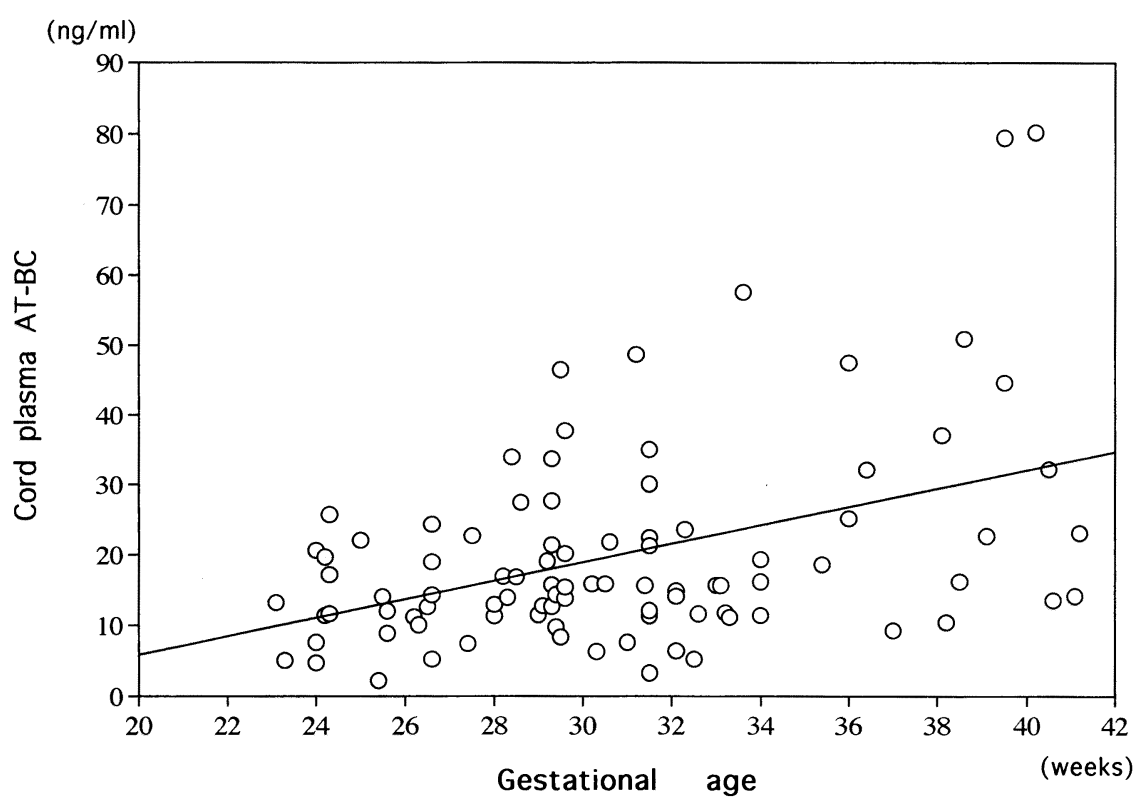

Fig. 1. A plot of cord plasma AT-BC against gestational age $(n=96)$. The regression line formula for all values indicated by a solid line, is a correlation coefficient $(r)$ of 0.428 and a $p<0.001$.

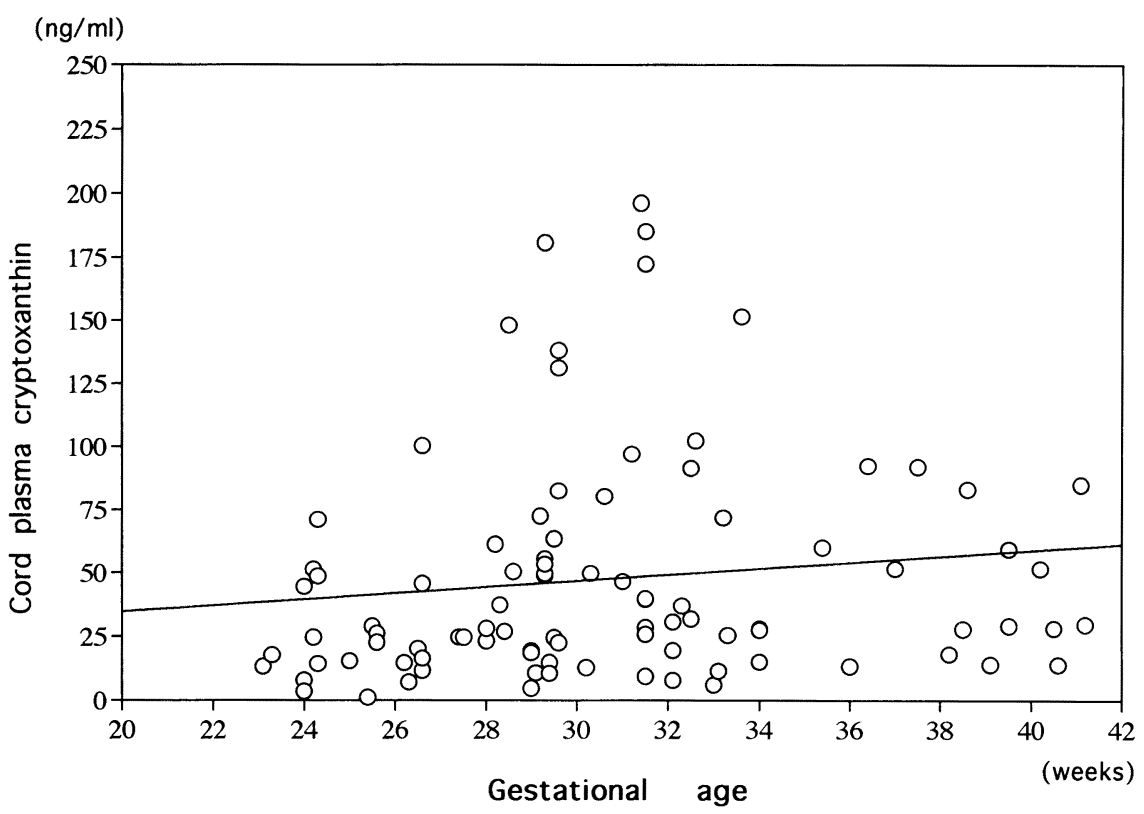

Fig. 2. A plot of cord plasma cryptoxanthin against gestational age $(n=96)$. The regression line formula for all values indicated by a solid line, is a correlation coefficient $(r)$ of 0.127 and a $p$ value of 0.225 . 


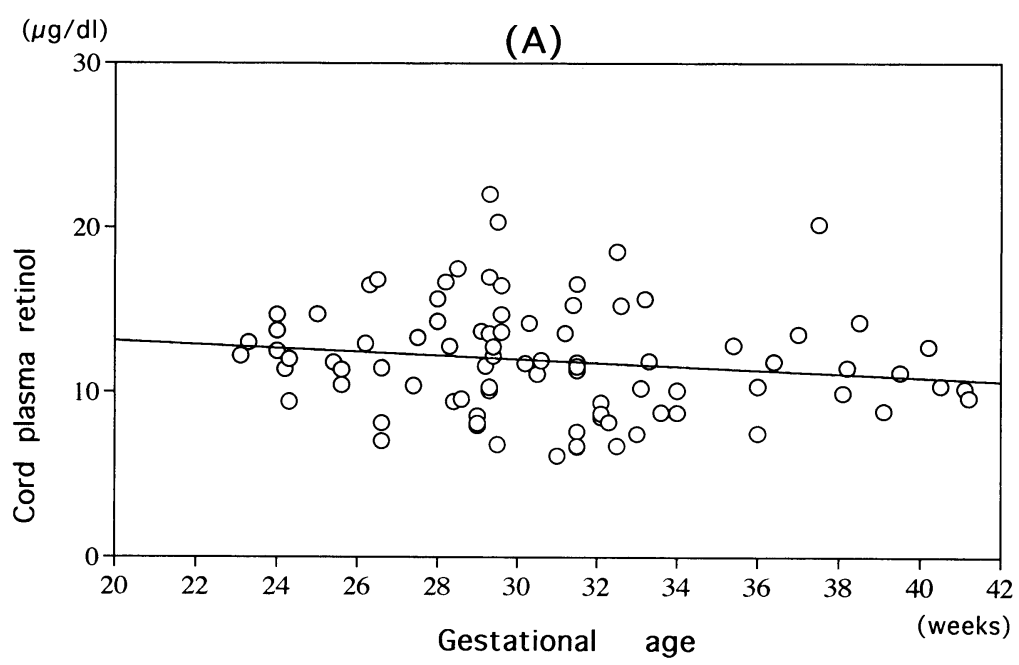

(B)

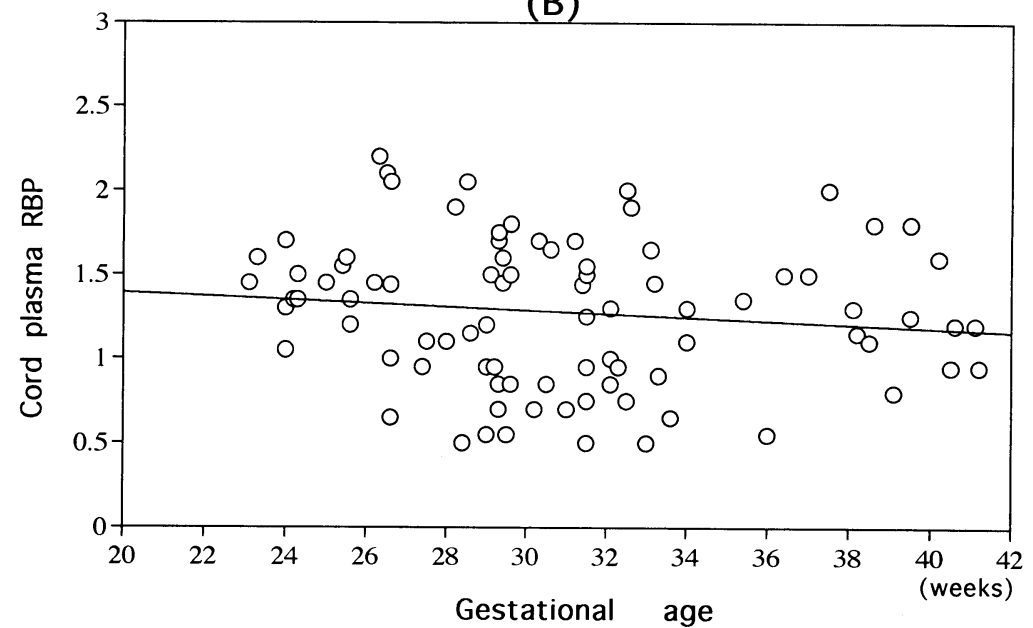

Fig. 3. A plot of cord plasma retinol (A) and RBP (B) against gestational age $(n=96)$. The regression line formula for all values indicated by a solid line, is a correlation coefficient $(r)$ of -0.144 and a $p$ value of 0.18 (retinol) and $(r)$ of -0.114 and a $p$ value of 0.29 (RBP).

neonates with toxemic mothers was significantly higher than that of neonates with nontoxemic mothers. No differences between the infants of mothers with and without toxemia were detected with respect to AT-BC, retinol, or RBP levels (Fig. 5). However, maternal plasma levels of AT-BC and cryptoxanthin were significantly decreased in toxemia, while retinol levels displayed no toxemia-related differences (Fig. 6). 

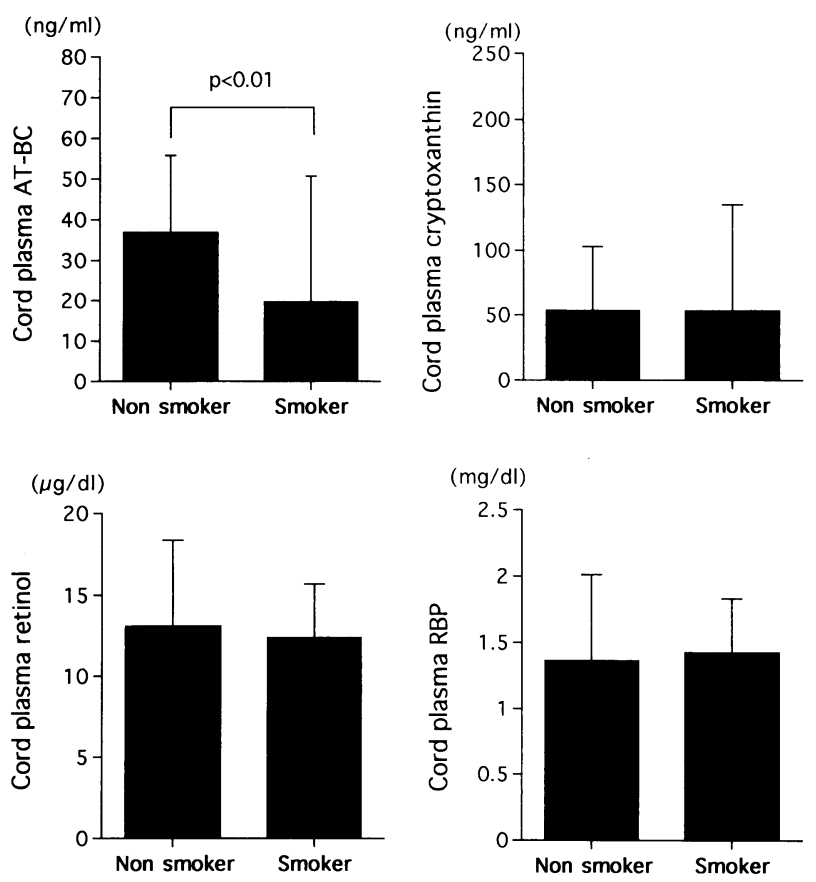

Fig. 4. Comparison of cord plasma concentrations of AT-BC, cryptoxanthin, retinol and RBP among mothers with smoking habits $(n=11)$ and without smoking habits $(n=56)$.

\section{Cord plasma vitamin levels and neonatal conditions}

The influence of perinatal conditions on plasma vitamin levels in cord blood was also examined. There were no differences with respect to cord plasma levels of AT-BC, cryptoxanthin, retinol and RBP between infants born to mothers with and without intrauterine infections (IUI) (Table 2). There was also no difference in this respect between infants with and without retinopathy of prematurity (Table 2), although retinol and its precursors may be related to visual development and function. Infants with retinopathy were defined as those who underwent therapeutic photocoagulation. Intrauterine growth retardation (IUGR) influenced only the levels of cryptoxanthin, which was higher in IUGR infants than in appropriate-fordates infants (Fig. 7), while no associated differences were recognized with respect to AT-BC, retinol or RBP levels. Although the pathogenesis and course of CLD have been reported to be related to retinol status in neonates $(3,7)$, there was no difference between infants with and without CLD as regards the cord blood levels of these vitamins (Table 3). There was also no difference in the cord blood vitamin levels of infants with respect to the micro bubble test applied to gastric aspirates in infants, which reflects the incidence of respiratory distress syndrome (RDS) after birth (Table 3). 

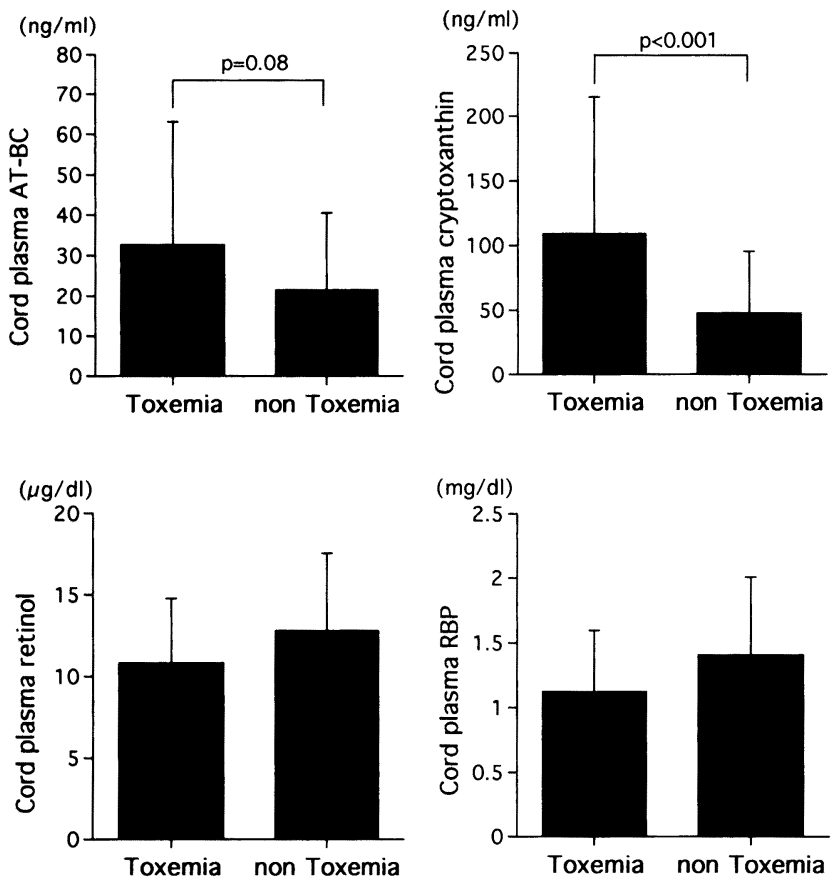

Fig. 5. Comparison of cord plasma concentrations of AT-BC, cryptoxanthin, retinol and RBP between toxemia $(n=12)$ and non toxemia $(n=55)$.
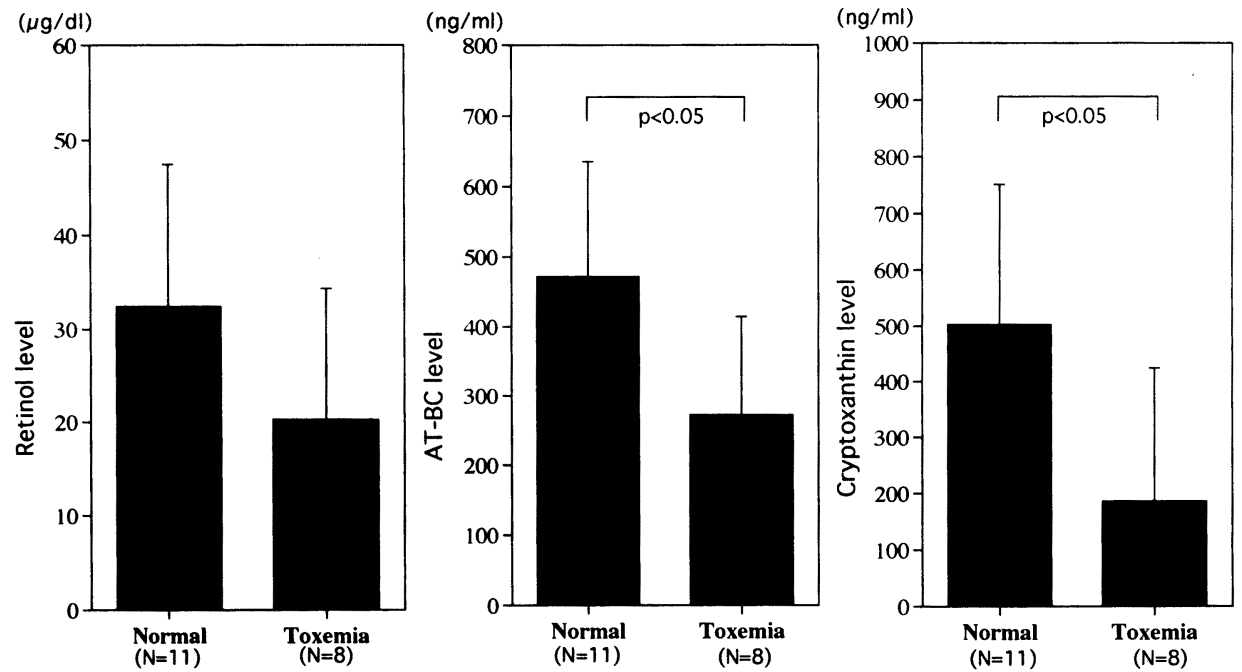

Fig. 6. Comparison of maternal blood concentrations of retinol, AT-BC and cryptoxanthin between mother with toxemia $(n=8)$ and without it $(n=11)$. 
Table 2. AT-BC, cryptoxanthin, retinol and RBP concentrations in cord blood from infants with intrauterine infections (IUI) or therapeutic photocoagulation for retinopathy of prematurity (ROP) and without them.

\begin{tabular}{lccccc}
\hline & $\begin{array}{c}\text { Intrauterine } \\
\text { infection } \\
(n=40)\end{array}$ & $\begin{array}{c}\text { Non-intrauterine } \\
\text { infection } \\
(n=56)\end{array}$ & & $\begin{array}{c}\text { Operation } \\
(+)^{*} \\
(n=5)\end{array}$ & $\begin{array}{c}\text { Operation } \\
(-)^{*} \\
(n=17)\end{array}$ \\
\cline { 2 - 3 } & $\mathrm{M} \pm \mathrm{SD}$ & $\mathbf{M} \pm \mathrm{SD}$ & & $\mathrm{M} \pm \mathrm{SD}$ & $\mathrm{M} \pm \mathrm{SD}$ \\
\hline AT-BC $(\mathrm{ng} / \mathrm{ml})$ & $16.5 \pm 8.7$ & $23.6 \pm 20.1$ & & $11.7 \pm 5.7$ & $13.8 \pm 6.9$ \\
Cryptoxanthin $(\mathrm{ng} / \mathrm{ml})$ & $48.0 \pm 40.7$ & $55.8 \pm 53.9$ & & $18.8 \pm 16.5$ & $30.2 \pm 25.7$ \\
Retinol $(\mu \mathrm{g} / \mathrm{dl})$ & $13.4 \pm 5.6$ & $12.8 \pm 6.3$ & & $12.7 \pm 2.6$ & $12.0 \pm 2.6$ \\
RBP $(\mathrm{mg} / \mathrm{dl})$ & $1.47 \pm 0.7$ & $1.43 \pm 1.1$ & & $1.48 \pm 0.4$ & $1.46 \pm 0.6$ \\
\hline
\end{tabular}

* Operation $(+)$ represents patients with therapeutic photocoagulation for retinopathy of prematurity and operation $(-)$ represents patients without it.
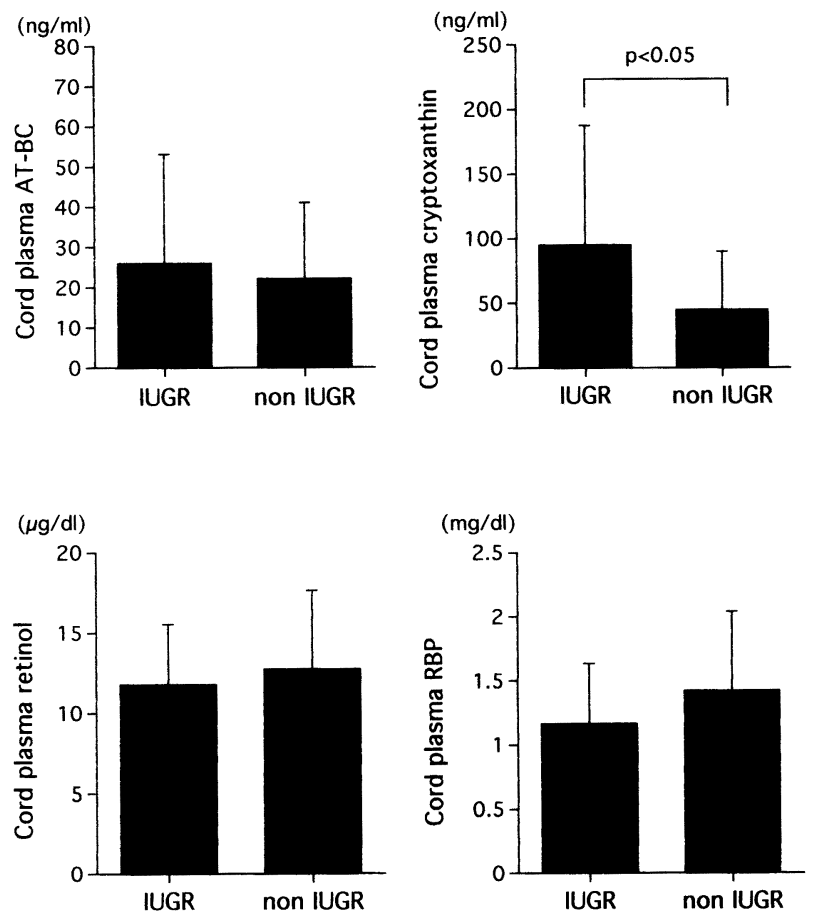

Fig. 7. Comparison of cord plasma concentrations of AT-BC, cryptoxanthin, retinol and RBP between IUGR $(n=18)$ and non IUGR $(n=65)$. 
Table 3. AT-BC, cryptoxanthin, retinol and RBP concentrations in cord blood from infants with chronic lung disease (CLD) and without it, and from infants among strong, moderate-weak and 0 in micro bubble test of gastric aspirate in them.

\begin{tabular}{|c|c|c|c|c|c|}
\hline & \multirow[b]{2}{*}{$\begin{array}{l}\text { CLD } \\
(n=6)\end{array}$} & \multirow{2}{*}{$\begin{array}{c}\text { Non-CLD } \\
(n=10)\end{array}$} & \multicolumn{3}{|c|}{ Stable micro bubble ratio } \\
\hline & & & $\begin{array}{l}\text { Strong } \\
(n=18)\end{array}$ & $\begin{array}{l}\text { Moderate-weak } \\
\quad(n=26)\end{array}$ & $\begin{array}{c}0 \\
(n=7)\end{array}$ \\
\hline & $\mathrm{M} \pm \mathrm{SD}$ & $\mathrm{M} \pm \mathrm{SD}$ & $\mathrm{M} \pm \mathrm{SD}$ & $\mathrm{M} \pm \mathrm{SD}$ & $\mathrm{M} \pm \mathrm{SD}$ \\
\hline AT-BC & $13.6 \pm 5.9$ & $17.9 \pm 8.4$ & $16.5 \pm 13.5$ & $23.7 \pm 20.6$ & $16.7 \pm 6.8$ \\
\hline Cryptoxanthin $(\mathrm{ng} / \mathrm{ml})$ & $32.7 \pm 30.9$ & $44.5 \pm 39.2$ & $40.2 \pm 40.1$ & $49.4 \pm 39.5$ & $75.2 \pm 70.5$ \\
\hline Retinol $(\mu \mathrm{g} / \mathrm{dl})$ & $12.8 \pm 2.3$ & $12.9 \pm 4.0$ & $11.8 \pm 3.4$ & $13.5 \pm 4.6$ & $11.9 \pm 3.1$ \\
\hline $\mathrm{RBP}(\mathrm{mg} / \mathrm{dl})$ & $1.62 \pm 0.5$ & $1.28 \pm 0.6$ & $1.30 \pm 0.4$ & $1.52 \pm 0.7$ & $1.20 \pm 0.5$ \\
\hline
\end{tabular}

In micro bubble test, the number of bubbles depends on the production of surfactants of lung. Cord blood levels of vitamins were classified by the number of bubbles; strong: $>20$, moderate-weak: 19-1, 0: nothing.

\section{DISCUSSION}

Brandt, et al. (14) investigated serum retinol levels in cord blood and reported that these levels were lower in premature infants than in term infants. In our study, plasma retinol and RBP levels in the cord blood of preterm infants were nearly equal to those of term infants, regardless of gestational age. Thus, our findings disagreed with those of Brandt, but agreed with those of Niwat (5). Nevertheless, our finding of very low cord blood levels of retinol was consistent with both previous reports $(5,14)$. Although some carotenoids including AT-BC and cryptoxanthin are precursors of vitamin A, there have been few investigations on their nutritional significance in the field of neonatal medicine $(15,16)$. It may be worth noting that epidemiologic studies of aging populations have demonstrated the role of carotenoids in reducing risks for cancer and degenerative diseases $(10,11)$. This is attributed to the action of carotenoids as potent oxygen radical quenchers and/or antioxidants (9). Carotenoid nutrition determined from plasma levels has been reported to be superior in breast-fed as compared with formula-fed infants, owing to the greater content of carotenoids in the colostrum of human milk as compared with that in powdered cow's milk (16). However, its nutritional significance in the neonatal period has not been established yet. Transport of carotenoids (as well as tocopherol) is poor $(15,16)$. AT-BC displayed plasma levels linearly increasing with gestational week, while cryptoxanthin did not, although both of these carotenoids are precursors of vitamin A. These carotenoids may have different biological significance during fetal and neonatal development, whereas plasma retinol and RBP levels in cord blood displayed no changes during gestation. Since, to our knowledge, there have been no reports on gestational changes in plasma carotenoids, the physiological significance of changes in AT-BC and cryptoxanthin status 
in fetuses and newborns has remained unexplained. Accordingly, we have investigated changes in cord blood carotenoid levels in relation to various maternal and infantile attributes, including maternal smoking habits and toxemia as well as intrauterine infections, IUGR, premature retinopathy, RDS and CLD. Maternal smoking significantly reduced plasma AT-BC levels in cord blood, but did not affect the levels of cryptoxanthin, retinol or RBP. Although it is generally known that the plasma $\beta$-carotene levels of smokers themselves are decreased $(8,12)$, our data show that maternal smoking also reduces fetal $\beta$-carotene status. Smoking is known to predispose mothers to toxemia and premature birth. Accordingly, plasma levels of retinol and carotenoids in cord blood were examined in relation to maternal toxemia. Cord blood from toxemic mothers displayed a higher plasma cryptoxanthin level than that from non-toxemic mothers, and AT-BC levels also tended to be higher in toxemic than in non-toxemic mothers (although this difference was not statistically significant), while retinol and RBP levels were unaffected by maternal toxemia. Thus, the changes in carotenoid levels were surprisingly different from those in retinol levels under the above conditions, but the reasons for this difference are not yet clearly understood. A higher plasma level of cryptoxanthin was also observed in the cord blood of infants with IUGR, but IUGR displayed no effects upon AT-BC, retinol, or RBP. As regards maternal smoking, tobacco smoke components which reduce plasma carotene may cross the placenta and impair fetal carotenoid status. Likewise, in maternal toxemia, toxic substances reducing plasma cryptoxanthin may be transferred to the fetus across the placenta, and/or other toxic substances transferred across the placenta could inhibit cryptoxanthin metabolism. Thus, disease conditions such as maternal toxemia and IUGR may lead to metabolic deterioration in the fetus and thereby impair utilization and metabolism of some carotenoids. Although several previous reports have indicated that cord blood levels of retinol and carotenoids are to some extent correlated with maternal blood levels $(15,16)$, our results disagreed with previous findings, since carotenoid levels associated with maternal toxemia in the present study were different between mother and fetus. Since 1980, numerous studies have been devoted to vitamin A nutritional status in the field of neonatal medicine. Particular attention has been devoted to the orderly growth and differentiation of epithelial cells in the respiratory tracts in connection with $\operatorname{CLD}(7,13)$, since severe retinol deficiency results in pathological changes in the tracheobronchial tree, including basal cell proliferation leading to necrosis of the lining epithelium and followed by squamous metaplasia. Necrotizing bronchiolitis and squamous metaplasia of the epithelium of the airways are frequently seen in infants dying of CLD following ventilatory management of conditions such as neonatal respiratory distress syndrome (3). In addition to previous evidence that plasma retinol levels are lower in premature infants than in term infants $(1,2,4)$, plasma retinol levels have also been investigated in CLD and are reported to be lower in infants with CLD than in those without CLD (3). Shenai et al. have treated VLBW and ELBW infants with retinol and reported that retinol may prevent the development of CLD (6). These findings suggest that poor 
vitamin A nutritional status in premature infants may be associated with the development of CLD after injury caused by hyperoxia and/or hyperventilation. Adequate carotenoid status in neonates could possibly prevent the development of neonatal CLD, as mentioned previously, and also that of retinopathy. No difference between infants with and without later CLD or between infants with and without retinopathy was noted with respect to carotenoid and retinol levels in the cord blood. Further studies are necessary to clarify the metabolic and functional significance of carotenoids as well as their conversion to retinol under disease conditions in mothers and fetuses.

\section{REFERENCES}

1) Bhatia, J., and Ziegler, E. E. (1983): Retinol-binding protein and prealbumin in cord blood of term and preterm infants. Early Hum. Dev., 8, 129-133.

2) Navarro, J., Causse, M. B., Desquilbet, N., Herve, F., and Lallemand, D. (1984): The vitamin status of low birth weight infants and their mothers. J. Pediatr. Gastroenterol. Nutr., 3, 744-748.

3) Shenai, J. P., Chytil, F., and Stahlman, M. T. (1985): Vitamin A status of neonates with bronchopulmonary dysplasia. Pediatr. Res., 19, 185-189.

4) Shenai, J. P., Chytil, F., Jhaveri, A., and Stahlman, M. T. (1981): Plasma vitamin A and retinol-binding protein in premature and term neonates. J. Pediatr., 99, 302-305.

5) Niwat, M., and James, A. O. (1979): Serum and liver concentrations of vitamin A in Thai fetuses as a function of gestational age. Am. J. Clin. Nutr., 32, 601-606.

6) Shenai, J. P., Kennedy, K. A., Chytil, F., et al. (1987): Clinical trial of vitamin A supplementation in infants susceptible to bronchopulmonary dysplasia. J. Pediatr., 111, 269-277.

7) Stern, L. (1979): The role of respirators in the etiology and pathogenesis of bronchopulmonary dysplasia. J. Pediatr., 95, 867-869.

8) Stryker, W. S., Kaplan, L. A., Stein, E. A., Stampfer, M. J., Sober, A., and Willett, W. C. (1988): The relation of diet, cigarette smoking, and alcohol consumption to plasma beta-carotene and alpha-tocopherol levels. Am. J. Epidemiol., 127, 283-296.

9) Burton, G. W., and Ingold, K. U. (1984): $\beta$-Carotene: An unusual type of lipid antioxidant. Science, 224, 569-573.

10) Wald, N. J., Thompson, S. G., Densem, J. W., Boreham, J., and Bailey, A. (1988): Serum beta-carotene and subsequent risk of cancer: Results from the BUPA study. $\mathrm{Br}$. J. Cancer, 57, 428-433.

11) Peto, R., Doll, R., Buckley, J. D., and Sporn, M. B. (1981): Can dietary beta-carotene materially reduce human cancer rates? Nature, 290, 201-208.

12) Palan, P. R., Romney, S. L., Vermund, S. H., Mikhail, M. G., and Basu, J. (1989): Effects of smoking and oral contraception on plasma $\beta$-carotene levels in healthy women. Am. J. Obstet. Gynecol., 161, 881-885.

13) Hustead, V. A., Gutcher, G. R., Anderson, S. A., and Zachman, R. D. (1984): Relationship of vitamin A (retinol) status to lung disease in preterm infant. J. Pediatr., 105, 610-615.

14) Brandt, R. B., Mueller, D. G., and Schroeder, J. R. (1978): Serum vitamin A in premature and term neonates. J. Pediatr., 92, 101-104.

Vol. 41, No. 6, 1995 
15) Hussein, L., El-Shawarby, O., Elnaggar, B., and Abdelmegid, A. (1988): Serum vitamin $\mathrm{A}$ and carotene concentrations among Egyptian fullterm neonates in relation to maternal status. Internat. J. Vit. Nutr., 58, 139-145.

16) Ostrea, E. M., Balun, J. E., Winkler, R., and Poter, T. (1986): Influence of breast-feeding on the restoration of the low serum concentration of vitamin $\mathrm{E}$ and $\beta$-carotene in the newborn infant. Am. J. Obstet. Gynecol., 154, 1014-1017. 